\title{
Dispersion management in two-photon microscopy by using diffractive optical elements
}

\author{
Jorge Pérez-Vizcaíno, ${ }^{1}$ Omel Mendoza-Yero, ${ }^{1}$ Gladys Mínguez-Vega, ${ }^{1, *}$ Raúl Martínez-Cuenca, ${ }^{1}$ \\ Pedro Andrés, ${ }^{2}$ and Jesús Lancis ${ }^{1}$ \\ ${ }^{1}$ Institut de Noves Tecnologies de la Imatge (INIT), Universitat Jaume I, Castelló 12080, Spain \\ ${ }^{2}$ Departament d'Òptica, Universitat de València, Burjassot 46100, Spain \\ ${ }^{*}$ Corresponding author: gminguez@uji.es
}

Received November 30, 2012; revised January 3, 2013; accepted January 6, 2013; posted January 7, 2013 (Doc. ID 180996); published February 8, 2013

\begin{abstract}
We demonstrate efficient generation of wide-field fluorescence signals in two-photon microscopy exploiting diffractive optical elements and short pulses by using a dispersion-compensated beam delivery optics module. Computer-generated holograms are codified onto a phase-only spatial light modulator, which allows for arbitrary single-shot patterning of the sample. Spatiotemporal shaping of the pulse is mandatory to overcome spatial chirp and pulse-front tilt effects that spread both in space and time the irradiance patterns, thus limiting not only the spatial resolution but also the signal-to-noise ratio in two-photon microscopy. By using a multipass amplifier delivering $30 \mathrm{fs}, 0.8 \mathrm{~mJ}$ pulses at $1 \mathrm{kHz}$ repetition rate, we experimentally demonstrated arbitrary single-shot fluorescence irradiance patterns in Rhodamine B. (C) 2013 Optical Society of America
\end{abstract}

OCIS codes: $\quad 090.1970,180.4315,190.4180$.

Two- and three-dimensional images of living tissue are widely demanded in life sciences [1]. In this context, multiphoton absorption processes [2] allow for a selective excitation of fluorescence signals, providing a convenient way to acquire images with good quality. The probability of these nonlinear processes is extremely low because they rely on the absorption of two or more photons to excite a molecule to a certain vibrational state from which a fluorescence signal is emitted. Hence, the fluorescence emission is restricted to a small region of the laser focus, allowing not only high spatial resolution imaging deep into tissue [3] but also naturally axial resolution.

In this context, the laser focus traditionally is scanned across the live samples, which cause that two-photon imaging technique to suffer from time limitations on signal acquisition [4]. In fact, the time required to acquire a complete two-dimensional image of the whole field of view, depends, among other factors, on the response time of scanners. Although this drawback has been improved by using different strategies to increase the scanning speed, which include resonant mirror systems [5], acousto-optical devices [6], or polygon-mirror scanners [7], such strategies can reduce the dwell time per pixel and can compromise signal-to-noise ratio. In addition, by selectively scanning only points of interest rather than all pixels of the sample, very fast pixel-by-pixel scan processes have been reported $[8,9]$.

In recent years, an alternative approach has been introduced to spatially multiplex the laser focus and then to carry out multibeam scanning simultaneously. This allows a more efficient use of the available laser power to either speed up imaging or to increase sampling per frame. The generation of an array of laser foci can be performed by multiple beam splitters [10], spinning disks [11], or diffractive optical elements [12]. Arbitrary diffraction patterns created by programmable spatial light modulators (SLMs), however, clearly offer more flexibility and customizability than imprinted fixed elements. For instance, SLMs can be used for simultaneous photomanipulation of living samples in a variable and temporally dynamic manner and for the stimulation or inhibition of multiple neurons at once to achieve specific excitatory and inhibition effects $[4,13]$.

For short pulses under 100 fs, however, the irradiance patterns generated with SLMs appear naturally blurred. Apart from possible distortion effects resulting from wavefront aberrations or light scattering, this unwanted physical phenomenon mainly is attributed to the linear dependence of the scale of the Fraunhofer irradiance patterns with the wavelength of light usually known as spatial chirp. In fact, spatial chirp causes an increasing of the eccentricity of the focused spots in applications, such as real-time two-photon absorption microscopy [14] and multifocal material processing [15]. In addition, the pulse-front tilt due to the propagation time difference between pulses coming from the SLM at different transverse positions also stretches the pulse in time. This kind of temporal pulse stretching cannot be precompensated with the chirping of the original pulse like it can be done in the case of group-velocity dispersion broadening caused by the pass of the ultrashort pulse through the material of optical components.

To illustrate the impact of spatial chirp and pulse-front tilt on two-photon microscopy, consider the use of a diffraction grating of period $p_{0}$ to generate multifocal spots under pulsed light illumination of central wavelength $\lambda_{0}$. We assume that the laser pulse is described by the onedimensional electric field $U(x, t)=U_{0} \exp \left(-x^{2} / 4 \sigma_{x}^{2}\right)$ $\exp \left(-t^{2} / 4 \sigma_{t}^{2}\right)$, where $\sigma_{x}$ and $\sigma_{t}$ denote the root-meansquare (rms) width of the spatial and temporal intensity profiles, respectively. With the help of a high numerical aperture microscope objective of focal length $f_{0}$, the diffraction orders (denoted by $n$ ) are spatially distributed at the sample plane, showing a set of tightly focused spots. As a result of the angular dispersion of the grating, each spectral component is focused at a different transverse position, which results in an elongated light spot. That is, the higher $n$, the longer the foci in the transversal direction $x$. Also, the decrease in the available bandwidth 
at an individual point due to the lateral walk-off of the different spectral components results in the temporal stretching of the pulse. As far as we move into higher diffraction orders, the pulse width $\sigma_{t}$ becomes more stretched. In contrast, the number of fluorescence photons produced per second $F_{p s}$ in a two-photon absorption process, decreases with increasing pulse width in accordance with the expression $F_{p s} \propto 1 / \sigma_{t}$ [2]. In this example, the relative spatial and temporal broadening, with respect to the unaffected zero diffraction order, can be estimated roughly by the expressions [16] $\Delta x \cong$ $n f_{0} \lambda_{0}^{2} /\left(4 \pi c p_{0} \sigma_{t}\right)$ and $\Delta \tau \cong n \lambda_{0} \sigma_{x} / c p_{0}$, respectively, where $c$ is the velocity of light in vacuum. For instance, for the set of parameters $f_{0}=10 \mathrm{~mm}, \lambda_{0}=800 \mathrm{~nm}, p_{0}=$ $83.3 \mu \mathrm{m}$ (or $12 \mathrm{lp} / \mathrm{mm}$ ), $\sigma_{t}=12.7 \mathrm{fs}$, and $\sigma_{x}=5 \mathrm{~mm}$, it can be verified easily that, for the first diffraction order, $\Delta x=1.6 \mu \mathrm{m}$ and $\Delta t=160 \mathrm{fs}$, or $\Delta x / \sigma_{0}=\Delta t / \sigma_{t} \cong 12.6$, where $\sigma_{0}$ is the rms width of the zero diffraction order. Such realistic values for the spatial and the temporal pulse broadening make the previous diffraction grating (like other diffractive optical elements) unsuitable for applications in multifocal multiphoton microscopy, as previously noted by Sacconi et al. [17]. Hence, both spatial chirp and pulse-front tilt phenomena adversely affect image quality and fluorescence generation, limiting the spatial resolution and the signal-to-noise ratio.

In this Letter we experimentally show two-photon absorption and fluorescence emission in Rhodamine B (RB) with spatially and temporally well-resolved irradiance patterns. To create arbitrary irradiance patterns in $\mathrm{RB}$, computer-generated holograms (CGHs) were encoded onto a phase-only SLM. This allows for a dynamic and programmable optical setup with a great deal of flexibility and customizability. Spatial chirp and pulse-front tilt effects were corrected, at first order, by using an hybrid diffractive-refractive optical system that was tested experimentally in the reconstruction of dynamical CGHs [18] and the generation of second-harmonic irradiance patterns [19] with imprinted diffractive optical elements.

Figure 1 shows the proposed setup. A mode-locked Ti: sapphire laser (Femtosource, Femtolaser) provides the excitation source. The temporal width of the pulses is $30 \mathrm{fs}$ amplitude FWHM ( $\left.\sigma_{t}=12.7 \mathrm{fs}\right)$, and the central wavelength of the corresponding spectrum is $\lambda_{0}=$ $800 \mathrm{~nm}$ with a bandwidth of approximately $50 \mathrm{~nm}$. The maximum energy per pulse is $0.8 \mathrm{~mJ}$ at a $1 \mathrm{kHz}$ repetition rate. The average energy per pulse was adjusted with a half-wave plate and a polarizer, and the spatial beam width was fixed using a reflective beam expander. The ultrashort light pulses from the Ti:sapphire laser passes

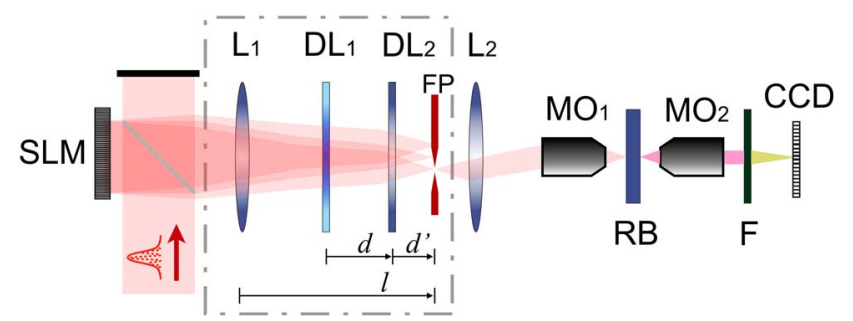

Fig. 1. (Color online) Schematic of the diffractive-refractive optical system used to improve two-photon absorption and fluorescence emission in RB. before exit through a postcompression stage based on fused silica Brewster prisms. In the beam delivery path, until the observation plane, one can introduce negative dispersion to later compensate for the positive material dispersion.

The pulsed laser beam impinges through a beam splitter onto a Fourier CGH encoded onto a phase-only SLM that, by virtue of the beam delivery optics (BDO) inside the dashed box, images the reconstructed hologram onto its back focal plane (FP). The CGHs are calculated by using the well-known Gerchberg-Saxton iterative Fourier transform algorithm but are carried out in two stages as proposed by Wyrowski [20]. The BDO is designed as a dispersion compensating module (DCM) to provide an output irradiance distribution corresponding to the nearly wavelength-independent Fourier transform of the hologram amplitude at its back FP. More specifically (see the dashed box in Fig. 1), beam delivery is accomplished by means of a lens achromat $L_{1}$ (with focal length $f_{1}=300 \mathrm{~mm}$ ) coupled to a diffractive lens pair $\mathrm{DL}_{1}, \mathrm{DL}_{2}$ as was introduced in [16]. A diffractive lens can be regarded as an optical element that focuses light by diffraction, following an inverse dependence with the wavelength of light. The focal lengths of $\mathrm{DL}_{1}$ and $\mathrm{DL}_{2}$ for the center wavelength of the laser are denoted by $f_{01}=$ $-150 \mathrm{~mm}$ and $f_{02}=150 \mathrm{~mm}$, respectively. Initially, the system acts as a Fourier transformer for the center wavelength, e.g., the field at FP is the Fourier transform of the field in the SLM plane for the center wavelength. In a second step, we force the design to ensure that reconstructed CGHs corresponding to each wavelength coalesces in a single spatial pattern for all the spectral components and that every ray impinging onto the SLM has an identical arrival time at the back FP. Although exact compensation is not possible, we impose a first-order, or achromatic, correction that leads to the geometrical constraints, $l=f_{1}, d^{2}=-f_{01} f_{02}$, and $d^{\prime}=-d^{2} /\left(d+2 f_{01}\right)$. To compare the optical features of our proposal with a conventional setup (without DCM), we simply remove the diffractive lens pair and displace the achromatic lens $L_{1}$ to locate its back focal plane at FP.

To excite the fluorescence signal in the $\mathrm{RB}$, we used a telescope composed of the refractive lens $L_{2}$ of focal length $f_{2}=100 \mathrm{~mm}$, and a $20 \times$ microscope objective $\mathrm{MO}_{1}$ of focal length $10 \mathrm{~mm}$. This telescope makes a reduced image of the irradiance pattern at the $\mathrm{RB}$, which is contained within a cubic glass box of $10 \mathrm{~mm}$ thickness. The additional relay optics preserves the spatiotemporal light distribution of the irradiance pattern except for the reduced spatial scale. To observe the fluorescence signal, the plane of $\mathrm{RB}$ is imaged onto a conventional CCD sensor (Ueye UI-1540M, $1280 \times 1024$ pixel resolution and 5.2 pixel pitch) by means of a $50 \times$ microscope objective $\mathrm{MO}_{2}$. We placed a suited filter F (BG39-Schott crystal) before the CCD camera to avoid transmission of infrared light.

In Fig. 2, results of the recorded fluorescence signal without (central column) and with (right column) the DCM are shown. In our experiment, the ability of the optical system to generate arbitrary irradiance distributions is illustrated with two examples, representing a bicycle in Figs. 2(a)-2(c), and a spiral in Figs. 2(d)-2(f). In the first column of Fig. 2 , computer reconstruction 

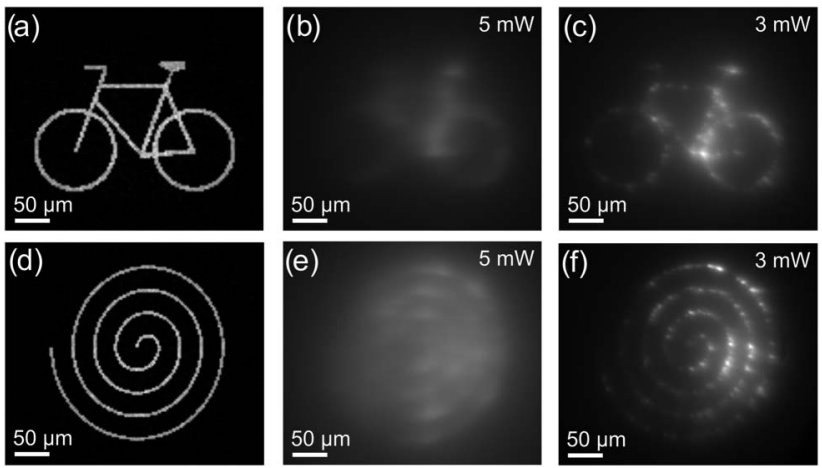

Fig. 2. Arbitrary fluorescence irradiance patterns obtained in RB (b) and (e) without compensation and (c) and (f) with DCM. (a) and (d) Corresponding reconstructed CGHs for the central wavelength of the laser.

of the CGHs corresponding to the bicycle Fig. 2(a) and the spiral Fig. 2(d) are shown. The computer reconstruction of the holograms spreads over an area of more than $400 \times 400$ pixels (or $2.08 \mathrm{~mm} \times 2.08 \mathrm{~mm}$ ). The spatial spectrum of the samples ranges from frequencies of around $25 \mathrm{lp} / \mathrm{mm}$ to about $38 \mathrm{lp} / \mathrm{mm}$. Within this interval, the effects of theoretical spatial and temporal broadening are calculated to vary from the values $\Delta x=3.3 \mu \mathrm{m}$, $\Delta t=330$ fs to $\Delta x=5 \mu \mathrm{m}, \Delta t=500 \mathrm{fs}$, respectively.

To ensure widefield fluorescence signaling all over the sample plane for the repetition rate and the pulse width of our laser system, the average power is adjusted to $3 \mathrm{~mW}$. As explained, patterning was accomplished through diffractive beam delivery. As reported in [17], we found out that spatial and temporal broadening of the pulse at the sample plane makes the use of diffractive optical element unsuitable, recording no signal. The fluorescence signal was subsequently recovered when the DCM was employed [see Figs. 2(c) and 2(f)]. We attribute this effect to the dispersion compensation capability of the DCM that preserves temporal width of the laser pulse at the sample plane, thus increasing the fluorescence signal with respect to the uncompensated situation. The number of emitted photons at the sample depends inversely on the pulse width. In addition, because of spatial chirp compensation, transverse spatial resolution for the CGH reconstruction is maintained. Finally, we check that diffraction-driven fluorescence signaling is possible without the DCM, but at the expense of using an additional $2 \mathrm{~mW}$ average power to compensate for temporal broadening. This is not recommended when using biological samples because high levels of excitation intensity may cause photodamage and photobleaching [3]. The uncompensated spatial chirp leads to a blurred signal that prevents correct hologram reconstruction and thus irradiance patterning; see Figs. $\underline{2(\mathrm{~b})}$ and $2(\mathrm{e})$.

This research was funded by the Spanish Ministerio de Ciencia e Innovación through Consolider Programme
(SAUUL CSD2007-00013) and project FIS2010-15746. Partial support from the Generalitat Valenciana and Fundació Caixa-Castelló through the projects PROMETEO/2012/021 and P11B2010-26 is also acknowledged. Authors are very grateful to the Serveis Centrals d'Intrumentació Científica (SCIC) of the Universitat Jaume I for the use of the femtosecond laser. We are indebted to Professor Jürgen Jahns from the FernUniversität Hagen (Germany) for making the DLs.

\section{References}

1. W. R. Zipfel, R. M. Williams, and W. W. Webb, Nat. Biotechnol. 21, 1369 (2003).

2. B. R. Masters, Handbook of Biomedical Nonlinear Optical Microscopy, B. R. Masters and P. T. C. So, eds. (Oxford University, 2008).

3. W. Denk, K. R. Delaney, A. Gelperin, D. Kleinfeld, B. W. Strowbridge, D. W. Tank, and R. Yuste, J. Neurosci. Methods 54, 151 (1994).

4. V. Nikolenko, B. O. Watson, R. Araya, A. Woodruff, D. S. Peterka, and R. Yuste, Front. Neural Circuits 2, 1 (2008).

5. N. L. Rochefort, O. Garaschuk, R.-I. Milos, M. Narushima, N. Marandi, B. Pichler, Y. Kovalchuk, and A. Konnerth, Proc. Natl. Acad. Sci. USA 106, 15049 (2009).

6. Y. Otsua, V. Bormutha, J. Wonga, B. Mathieub, G. P. Duguéa, A. Feltz, and S. Dieudonnéa, J. Neurosci. Methods 173, 259 (2008).

7. W. C. Warger, G. S. Laevsky, D. J. Townsend, M. Rajadhyaksha, and Ch. A. DiMarzio, J. Biomed. Opt. 12, 044006 (2007).

8. W. Göbel and F. Helmchen, J. Neurophysiol. 98, 3770 (2007).

9. K. P. Lillis, A. Enga, J. A. White, and J. Mertz, J. Neurosci. Methods 172, 178 (2008).

10. T. Nielsen, M. Fricke, D. Hellweg, and P. Andresen, J. Microsc. 201, 368 (2001).

11. J. Bewersdorf, R. Pick, and S. W. Hell, Opt. Lett. 23, 655 (1998).

12. D. C. O'Shea, T. J. Suleski, A. D. Kathman, and D. W. Prather, Diffractive Optics: Design, Fabrication, and Test (SPIE, 2004).

13. B. O. Watson, V. Nikolenko, R. Araya, D. S. Peterka, A. Woodruff, and R. Yuste, Front. clin. Neurosci. 4, 1 (2010).

14. A. H. Buist, M. Müller, J. Squer, and G. J. Brakenhoff, J. Microsc. 192, 217 (1998).

15. Z. Kuang, W. Perrie, J. Leach, M. Sharp, S. P. Edwardson, M. Padgett, G. Dearden, and K. G. Watkins, Appl. Surf. Sci. 255, 2284 (2008).

16. G. Mínguez-Vega, J. Lancis, J. Caraquitena, V. Torres-Company, and P. Andrés, Opt. Lett. 31, 2631 (2006).

17. L. Sacconi, E. Froner, R. Antolini, M. R. Taghizadeh, A. Choudhury, and F. S. Pavone, Opt. Lett. 28, 1918 (2003).

18. Ll. Martínez-León, P. Clemente, E. Tajahuerce, G. Mínguez-Vega, O. Mendoza-Yero, M. Fernández-Alonso, J. Lancis, V. Climent, and P. Andrés, Appl. Phys. Lett. 94, 011104 (2009).

19. R. Martínez-Cuenca, O. Mendoza-Yero, B. Alonso, Í. J. Sola, G. Mínguez-Vega, and J. Lancis, Opt. Lett. 37, 957 (2012).

20. F. Wyrowski, J. Opt. Soc. Am. A 7, 961 (1990). 